\title{
Existence of multiple solutions for fractional $p$-Kirchhoff equations with concave-convex nonlinearities
}

Libo Yang ${ }^{1,2^{*}}$ and Tianqing $A n^{1}$

\section{"Correspondence:}

yanglibo80@126.com

${ }^{1}$ College of Science, Hohai

University, Nanjing, 210098 ,

P.R. China

${ }^{2}$ Faculty of Mathematics and

Physics, Huaiyin Institute of Technology, Huai'an, 223003, P.R. China

\section{Springer}
Abstract
In this paper, we investigate the existence of multiple solutions for Kirchhoff-type equations involving nonlocal integro-differential operators with homogeneous Dirichlet boundary conditions as follows:

$$
\begin{cases}M\left(\int_{\mathbb{R}^{2 n}} \frac{|u(x)-u(y)|^{p}}{\left.|x-y|\right|^{n+s p}} d x d y\right)(-\triangle)_{p}^{s} u=\lambda|u|^{q-2} u+\frac{\alpha}{\alpha+\beta}|u|^{\alpha-2} u|v|^{\beta}, & \text { in } \Omega, \\ M\left(\int_{\mathbb{R}^{2 n}} \frac{|v(x)-v(y)|^{p}}{|x-y|^{\mid+s p}} d x d y\right)(-\triangle)_{p}^{s} v=\mu|v|^{a-2} v+\frac{\beta}{\alpha+\beta}|v|^{\beta-2} v|u|^{\alpha}, & \text { in } \Omega, \\ u=v=0, & \text { in } \mathbb{R}^{n} \backslash \Omega,\end{cases}
$$

where $\Omega$ is a smooth bounded set in $\mathbb{R}^{n}, n>p s$ with $s \in(0,1)$ fixed, $\lambda, \mu>0$ are two parameters, $1<q<p<p(\tau+1)<\alpha+\beta<p^{*}, p^{*}=\frac{n p}{n-s p}, M$ is a continuous function, given by $M(h)=k+I h^{\tau}, k>0, I, \tau \geq 0$, and $(-\triangle)_{p}^{S}$ is the fractional $p$-Laplacian operator. We will prove that the problem has at least two solutions by using the Nehari manifold method and fibering maps.

Keywords: Kirchhoff-type equations; fractional $p$-Laplacian; concave-convex nonlinearities; Nehari manifold method; fibering maps

\section{Introduction}

In this paper, we consider the following Kirchhoff-type problem involving fractional $p$ Laplacian and concave-convex nonlinearities:

$$
\begin{cases}M\left(\int_{\mathbb{R}^{2 n}} \frac{|u(x)-u(y)|^{p}}{|x-y|^{n+s p}} d x d y\right)(-\triangle)_{p}^{s} u=\lambda|u|^{q-2} u+\frac{\alpha}{\alpha+\beta}|u|^{\alpha-2} u|v|^{\beta}, & \text { in } \Omega, \\ M\left(\int_{\mathbb{R}^{2 n}} \frac{|v(x)-v(y)|^{p}}{|x-y|^{n+s p}} d x d y\right)(-\triangle)_{p}^{s} v=\mu|v|^{q-2} v+\frac{\beta}{\alpha+\beta}|v|^{\beta-2} v|u|^{\alpha}, & \text { in } \Omega, \\ u=v=0, & \text { in } \mathbb{R}^{n} \backslash \Omega,\end{cases}
$$

where $\Omega$ is a smooth bounded set in $\mathbb{R}^{n}, n>p s$ with $s \in(0,1)$ fixed, $\lambda, \mu>0$ are two parameters, $1<q<p<p(\tau+1)<\alpha+\beta<p^{*}, p^{*}=\frac{n p}{n-s p}$ is the fractional Sobolev exponent, $M$ is a special continuous function defined by $M(h)=k+l h^{\tau}, k>0, l, \tau \geq 0 .(-\triangle)_{p}^{s}$ is the fractional $p$-Laplacian operator given by

$$
(-\triangle)_{p}^{s} u(x)=2 \lim _{\varepsilon \rightarrow 0} \int_{\mathbb{R}^{n} \backslash B_{\varepsilon}(x)} \frac{|u(x)-u(y)|^{p-2}(u(x)-u(y))}{|x-y|^{n+s p}} d x d y .
$$

(0) The Author(s) 2017. This article is distributed under the terms of the Creative Commons Attribution 4.0 International License (http://creativecommons.org/licenses/by/4.0/), which permits unrestricted use, distribution, and reproduction in any medium, provided you give appropriate credit to the original author(s) and the source, provide a link to the Creative Commons license, and indicate if changes were made. 
The Kirchhoff-type equation and system have a broad background in phase transitions, population dynamics, mathematical finance, etc. There have been a lot of excellent results related to the existence and multiplicity of solutions for this system. We refer the readers to [1-4] for Kirchhoff problems involving the classical Laplace operator and to [5, 6] for the $p$-Laplacian case. For the fractional system, please consult [7-21] and the references therein.

In [10] and [11], the authors discussed the system (or a single equation, that is, $u=v$ ) in the special case of $M \equiv 1$. They obtained some interesting results by using the Nehari manifold method. For the special case $p=2$ of this system, there are many results available in the existing literature, we refer the interested reader to $[22,23]$ for the case of the classical Laplacian and to [24-26] for the case of the fractional Laplacian. Moreover, the authors [18] studied bifurcation results for a fractional elliptic equation with critical exponent. There is also some work for the case that $M$ is not a constant (see, for example, [9]). However, as far as we know, there are few results on the fractional $p$-Kirchhoff system with concave-convex nonlinearities. Motivated by the above work, in this paper we consider problem (1.1) for a more general case $M(h)=k+l h^{\tau}$. We obtained a new multiplicity result by using the Nehari manifold method and fibering maps.

In order to state our result, we introduce some notations. Suppose $s \in(0,1)$ and $p \in$ $[1, \infty)$. Let $W^{s, p}$ be a fractional Sobolev space with the norm

$$
\|u\|_{W^{s, p}(\Omega)}=\|u\|_{L^{p}(\Omega)}+\left(\int_{\Omega \times \Omega} \frac{|u(x)-u(y)|^{p}}{|x-y|^{n+s p}} d x d y\right)^{\frac{1}{p}} .
$$

Set $Q=\mathbb{R}^{2 n} \backslash(C \Omega \times C \Omega)$ with $C \Omega=\mathbb{R}^{n} \backslash \Omega$. We define

$$
X=\left\{u \mid u: \mathbb{R}^{n} \rightarrow \mathbb{R} \text { is measurable, }\left.u\right|_{\Omega} \in L^{p}(\Omega) \text {, and } \int_{Q} \frac{|u(x)-u(y)|^{p}}{|x-y|^{n+s p}} d x d y<\infty\right\} .
$$

The space $X$ is endowed with the norm

$$
\|u\|_{X}=\|u\|_{L^{p}(\Omega)}+\left(\int_{Q} \frac{|u(x)-u(y)|^{p}}{|x-y|^{n+s p}} d x d y\right)^{\frac{1}{p}} .
$$

Let $X_{0}$ be the completion of the space $C_{0}^{\infty}(\Omega)$ in $X$. The space $X_{0}$ is a Banach space which can be endowed with the norm

$$
\|u\|_{X_{0}}=\left(\int_{Q} \frac{|u(x)-u(y)|^{p}}{|x-y|^{n+s p}} d x d y\right)^{\frac{1}{p}}
$$

It is easy to see that this norm is equivalent to the usual one defined in (1.3).

As proved in $[17,24]$, we have the following results:

(i) $X_{0} \hookrightarrow L^{r}(\Omega)$ is continuous for any $r \in\left[1, p^{*}\right]$ and compact for any $r \in\left[1, p^{*}\right)$.

(ii) For $\alpha+\beta \in\left(p, p^{*}\right)$, let $S$ denote the best Sobolev constant for the embedding $X_{0} \hookrightarrow L^{\alpha+\beta}(\Omega)$. Then, for $u \in X_{0}$, we have

$$
\begin{aligned}
\|u\|_{L^{\alpha+\beta}(\Omega)} & =\left(\int_{\Omega}|u|^{\alpha+\beta} d x\right)^{\frac{1}{\alpha+\beta}} \leq S^{-\frac{1}{p}}\|u\|_{X_{0}} \\
& =S^{-\frac{1}{p}}\left(\int_{Q} \frac{|u(x)-u(y)|^{p}}{|x-y|^{n+s p}} d x d y\right)^{\frac{1}{p}} .
\end{aligned}
$$


Let $E=X_{0} \times X_{0}$ be the Cartesian product of two spaces, which is a reflexive Banach space with the norm

$$
\begin{aligned}
\|(u, v)\| & =\left(\|u\|_{X_{0}}^{p}+\|v\|_{X_{0}}^{p}\right)^{\frac{1}{p}} \\
& =\left(\int_{Q} \frac{|u(x)-u(y)|^{p}}{|x-y|^{n+s p}} d x d y+\int_{Q} \frac{|v(x)-v(y)|^{p}}{|x-y|^{n+s p}} d x d y\right)^{\frac{1}{p}} .
\end{aligned}
$$

Definition 1.1 We say that $(u, v) \in E$ is a weak solution of problem (1.1) if for any $(\phi, \psi) \in E$ one has

$$
\begin{aligned}
& M\left(\|u\|_{X_{0}}\right) \int_{Q} \frac{|u(x)-u(y)|^{p-2}(u(x)-u(y))(\phi(x)-\phi(y))}{|x-y|^{n+s p}} d x d y \\
& \quad+M\left(\|v\|_{X_{0}}\right) \int_{Q} \frac{|v(x)-v(y)|^{p-2}(v(x)-v(y))(\psi(x)-\psi(y))}{|x-y|^{n+s p}} d x d y \\
& =\int_{\Omega}\left(\lambda|u|^{q-2} u \phi+\mu|v|^{q-2} v \psi\right) d x+\frac{\alpha}{\alpha+\beta} \int_{\Omega}|u|^{\alpha-2} u|v|^{\beta} \phi d x \\
& \quad+\frac{\beta}{\alpha+\beta} \int_{\Omega}|u|^{\alpha}|v|^{\beta-2} v \psi d x .
\end{aligned}
$$

The main result of this paper is as follows.

Theorem 1.2 Let $s \in(0,1), n>s p$. If $1<q<p<p(\tau+1)<\alpha+\beta<p^{*}$, then there exists $\Lambda_{0}>0$ such that for $0<\lambda+\mu<\Lambda_{0}$ problem (1.1) has at least two solutions.

Remark 1 To our best knowledge, there is no similar result of this system for the case $p=2$.

This paper is organized as follows. In Section 2, we give some preliminaries of a Nehari manifold and a variational setting of problem (1.1). Section 3 gives the proof of Theorem 1.2.

\section{The variational setting}

Define a functional $I(u, v): E \rightarrow \mathbb{R}$ as follows:

$$
I(u, v)=\frac{k}{p}\|(u, v)\|^{p}+\frac{l}{\sigma}\|(u, v)\|^{\sigma}-\frac{1}{m} \int_{\Omega}|u|^{\alpha}|v|^{\beta} d x-\frac{1}{q} G(u, v),
$$

where $\sigma=p(\tau+1)$, and $m=\alpha+\beta$, and

$$
G(u, v)=\int_{\Omega}\left(\lambda|u|^{q}+\mu|v|^{q}\right) d x .
$$

By a direct computation, we know that $I(u, v) \in C^{1}(E, \mathbb{R})$ and, for $\forall(\phi, \psi) \in E$, there holds

$$
\begin{aligned}
\left\langle I^{\prime}(u, v),(\phi, \psi)\right\rangle= & M\left(\|u\|_{X_{0}}\right) \int_{Q} \frac{|u(x)-u(y)|^{p-2}(u(x)-u(y))(\phi(x)-\phi(y))}{|x-y|^{n+s p}} d x d y \\
& +M\left(\|v\|_{X_{0}}\right) \int_{Q} \frac{|v(x)-v(y)|^{p-2}(v(x)-v(y))(\psi(x)-\psi(y))}{|x-y|^{n+s p}} d x d y
\end{aligned}
$$




$$
\begin{aligned}
& -\int_{\Omega}\left(\lambda|u|^{q-2} u \phi+\mu|v|^{q-2} \nu \psi\right) d x-\frac{\alpha}{m} \int_{\Omega}|u|^{\alpha-2} u|v|^{\beta} \phi d x \\
& -\frac{\beta}{m} \int_{\Omega}|u|^{\alpha}|v|^{\beta-2} \nu \psi d x .
\end{aligned}
$$

Then the weak solutions of problem (1.1) correspond to the critical points of the functional $I$. Since $I$ is not bounded below on $E$, we consider it on the Nehari manifold

$$
\left.N=\left\{(u, v) \in E \backslash(0,0)|| I^{\prime}(u, v),(u, v)\right\rangle=0\right\} .
$$

From (2.2), we have

$$
\left\langle I^{\prime}(u, v),(u, v)\right\rangle=k\|(u, v)\|^{p}+l\|(u, v)\|^{\sigma}-\int_{\Omega}|u|^{\alpha}|v|^{\beta} d x-G(u, v) .
$$

Thus, $(u, v) \in N$ if and only if

$$
k\|(u, v)\|^{p}+l\|(u, v)\|^{\sigma}-\int_{\Omega}|u|^{\alpha}|v|^{\beta} d x-G(u, v)=0 .
$$

Particularly, the following equality holds on $N$ :

$$
\begin{aligned}
I(u, v) & =k\left(\frac{1}{p}-\frac{1}{q}\right)\|(u, v)\|^{p}+l\left(\frac{1}{\sigma}-\frac{1}{q}\right)\|(u, v)\|^{\sigma}-\left(\frac{1}{m}-\frac{1}{q}\right) \int_{\Omega}|u|^{\alpha}|v|^{\beta} d x \\
& =k\left(\frac{1}{p}-\frac{1}{m}\right)\|(u, v)\|^{p}+l\left(\frac{1}{\sigma}-\frac{1}{m}\right)\|(u, v)\|^{\sigma}-\left(\frac{1}{q}-\frac{1}{m}\right) G(u, v) .
\end{aligned}
$$

Define

$$
\Phi(u, v)=\left\langle I^{\prime}(u, v),(u, v)\right\rangle, \quad \forall(u, v) \in E .
$$

Then, for any $(u, v) \in N$,

$$
\begin{aligned}
& \left\langle\Phi^{\prime}(u, v),(u, v)\right\rangle \\
& \quad=k p\|(u, v)\|^{p}+l \sigma\|(u, v)\|^{\sigma}-m \int_{\Omega}|u|^{\alpha}|v|^{\beta} d x-q G(u, v) \\
& =k(p-m)\|(u, v)\|^{p}+l(\sigma-m)\|(u, v)\|^{\sigma}-(q-m) G(u, v) \\
& =k(p-q)\|(u, v)\|^{p}+l(\sigma-q)\|(u, v)\|^{\sigma}-(m-q) \int_{\Omega}|u|^{\alpha}|v|^{\beta} d x .
\end{aligned}
$$

Thus, it is natural to split $N$ into three parts:

$$
\begin{aligned}
& N^{+}=\left\{(u, v) \in N:\left\langle\Phi^{\prime}(u, v),(u, v)\right\rangle>0\right\}, \\
& N^{-}=\left\{(u, v) \in N:\left\langle\Phi^{\prime}(u, v),(u, v)\right\rangle<0\right\}, \\
& N^{0}=\left\{(u, v) \in N:\left\langle\Phi^{\prime}(u, v),(u, v)\right\rangle=0\right\} .
\end{aligned}
$$

We now derive some properties of $N^{+}, N^{-}$and $N^{0}$. 
Lemma 2.1 I is coercive and bounded below on $N$.

Proof By Hölder's inequality and (1.6), we have

$$
\begin{aligned}
\int_{\Omega} \lambda|u|^{q} d x & \leq \lambda\left(\int_{\Omega} 1 d x\right)^{\frac{m-q}{m}}\left(\int_{\Omega}|u|^{m} d x\right)^{\frac{q}{m}}=\lambda|\Omega|^{\frac{m-q}{m}}\|u\|_{m}^{q} \\
& \leq \lambda|\Omega|^{\frac{m-q}{m}} S^{-\frac{q}{p}}\|u\|_{X_{0}}^{q} \leq \lambda|\Omega|^{\frac{m-q}{m}} S^{-\frac{q}{p}}\|(u, v)\|^{q} .
\end{aligned}
$$

Similarly,

$$
\int_{\Omega} \mu|v|^{q} d x \leq \mu|\Omega|^{\frac{m-q}{m}} S^{-\frac{q}{p}}\|v\|_{X_{0}}^{q} \leq \mu|\Omega|^{\frac{m-q}{m}} S^{-\frac{q}{p}}\|(u, v)\|^{q} .
$$

Then

$$
G(u, v) \leq(\lambda+\mu)|\Omega|^{\frac{m-q}{m}} S^{-\frac{q}{p}}\|(u, v)\|^{q} .
$$

It follows from (2.5) and (2.8) that

$$
\begin{aligned}
I(u, v) \geq & k\left(\frac{1}{p}-\frac{1}{m}\right)\|(u, v)\|^{p}+l\left(\frac{1}{\sigma}-\frac{1}{m}\right)\|(u, v)\|^{\sigma} \\
& -\left(\frac{1}{q}-\frac{1}{m}\right)(\lambda+\mu)|\Omega|^{\frac{m-q}{m}} S^{-\frac{q}{p}}\|(u, v)\|^{q} .
\end{aligned}
$$

Since $q<p \leq \sigma<m$, from inequality (2.9), the functional $I$ is coercive and bounded below on $N$. The proof is completed.

Lemma 2.2 There exists $\Lambda_{0}>0$, given by

$$
\Lambda_{0}=\frac{k(m-p)}{(m-q)|\Omega|^{\frac{m-q}{m}} S^{-\frac{q}{p}}}\left(\frac{k(p-q)}{(m-q) S^{-\frac{m}{q}}}\right)^{\frac{p-q}{m-p}},
$$

such that for any $0<\lambda+\mu<\Lambda_{0}$ we have $N^{0}=\emptyset$.

Proof We argue by contradiction. Assume that there exist $\lambda$, $\mu>0$ with $0<\lambda+\mu<\Lambda_{0}$ such that $N^{0} \neq \emptyset$. Then, for $(u, v) \in N^{0}$, we have

$$
\left\langle I^{\prime}(u, v),(u, v)\right\rangle=0 \quad \text { and } \quad\left\langle\Phi^{\prime}(u, v),(u, v)\right\rangle=0 .
$$

Then it follows from (2.5)-(2.8) that

$$
\|(u, v)\| \leq\left(\frac{(m-q)(\lambda+\mu)|\Omega|^{\frac{m-q}{m}} S^{-\frac{q}{p}}}{k(m-p)}\right)^{\frac{1}{p-q}} .
$$

On the other hand, by Young's inequality, we have

$$
\begin{aligned}
\int_{\Omega}|u|^{\alpha}|v|^{\beta} d x & \leq \frac{\alpha}{m} \int_{\Omega}|u|^{m} d x+\frac{\beta}{m} \int_{\Omega}|v|^{m} d x \\
& \leq \frac{\alpha}{m} S^{-\frac{m}{q}}\|u\|_{X_{0}}^{m}+\frac{\beta}{m} S^{-\frac{m}{q}}\|v\|_{X_{0}}^{m} \leq S^{-\frac{m}{q}}\|(u, v)\|^{m} .
\end{aligned}
$$


From (2.5)-(2.7) and (2.11) it follows that

$$
k(p-q)\|(u, v)\|^{p} \leq(m-q) S^{-\frac{m}{q}}\|(u, v)\|^{m} .
$$

We have

$$
\|(u, v)\| \geq\left(\frac{k(p-q)}{(m-q) S^{-\frac{m}{q}}}\right)^{\frac{1}{m-p}}
$$

By (2.10) and (2.12),

$$
\lambda+\mu \geq \frac{k(m-p)}{(m-q)|\Omega|^{\frac{m-q}{m}} S^{-\frac{q}{p}}}\left(\frac{k(p-q)}{(m-q) S^{-\frac{m}{q}}}\right)^{\frac{p-q}{m-p}}=\Lambda_{0},
$$

which contradicts $0<\lambda+\mu<\Lambda_{0}$.

By Lemmas 2.1 and 2.2, we write $N=N^{+}+N^{-}$for $0<\lambda+\mu<\Lambda_{0}$, and $I$ is coercive and bounded from below on $N^{+}$and $N^{-}$. We define

$$
C^{+}=\inf _{(u, v) \in N^{+}} I(u, v), \quad C^{-}=\inf _{(u, v) \in N^{-}} I(u, v) .
$$

As proved in [27], we have the following lemma.

Lemma 2.3 For $0<\lambda+\mu<\Lambda_{0}$, suppose that $\left(u_{0}, v_{0}\right)$ is a local minimizer for I on $N$. Then, if $\left(u_{0}, v_{0}\right) \notin N^{0},\left(u_{0}, v_{0}\right)$ is a critical point of $I$.

\section{Lemma 2.4}

(a) If $0<\lambda+\mu<\Lambda_{0}$, then $C^{+}<0$.

(b) If $0<\lambda+\mu<\frac{q}{p} \Lambda_{0}$, then $\exists d_{0}>0$ such that $C^{-}>d_{0}$.

Proof (a) Let $(u, v) \in N^{+}$, it follows from (2.6) and (2.7) that

$$
\int_{\Omega}|u|^{\alpha}|v|^{\beta} d x<\frac{k(p-q)}{m-q}\|(u, v)\|^{p}+\frac{l(\sigma-q)}{m-q}\|(u, v)\|^{\sigma} .
$$

Put (2.13) into (2.5),

$$
I(u, v)<-\frac{k(p-q)}{m p q}\|(u, v)\|^{p}-\frac{l(p-q)(m-p)}{m p q}\|(u, v)\|^{\sigma}<0,
$$

which implies $C^{+}=\inf _{(u, v) \in N^{+}} I(u, v)<0$.

(b) Let $(u, v) \in N^{-}$. By (2.5) and (2.8),

$$
\begin{aligned}
I(u, v) & \geq \frac{k(m-p)}{p m}\|(u, v)\|^{p}-\frac{m-q}{m q} G(u, v) \\
& \geq \frac{k(m-p)}{p m}\|(u, v)\|^{p}-\frac{m-q}{m q}(\lambda+\mu)|\Omega|^{\frac{m-q}{m}} S^{-\frac{q}{p}}\|(u, v)\|^{q} \\
& =\|(u, v)\|^{q}\left(\frac{k(m-p)}{p m}\|(u, v)\|^{p-q}-\frac{m-q}{m q}(\lambda+\mu)|\Omega|^{\frac{m-q}{m}} S^{-\frac{q}{p}}\right) .
\end{aligned}
$$


Combining (2.12) with (2.14), we have

$$
I(u, v) \geq\left(\frac{k(p-q)}{(m-q) S^{-\frac{m}{q}}}\right)^{\frac{q}{m-p}}\left(\frac{k(m-p)}{p m}\left(\frac{k(p-q)}{(m-q) S^{-\frac{m}{q}}}\right)^{\frac{p-q}{m-p}}-\frac{m-q}{m q}(\lambda+\mu)|\Omega|^{\frac{m-q}{m}} S^{-\frac{q}{p}}\right) .
$$

Clearly, if $0<\lambda+\mu<\Lambda_{0}$, then there exists $d_{0}(p, q, \alpha, \beta, S)>0$ such that $C^{-}=\inf _{(u, v) \in N^{-}} I(u$, v) $>d_{0}$.

For each $(u, v) \in E$, let

$$
\eta(t)=k t^{p-q}\|(u, v)\|^{p}+l t^{\sigma-q}\|u, v\|^{\sigma}-t^{m-q} \int_{\Omega}|u|^{\alpha}|v|^{\beta} d x .
$$

Then

$$
\eta^{\prime}(t)=t^{p-q-1} E(t)
$$

where

$$
E(t)=k(p-q)\|(u, v)\|^{p}+l(\sigma-q) t^{\sigma-p}\|(u, v)\|^{\sigma}-(m-q) t^{m-p} \int_{\Omega}|u|^{\alpha}|v|^{\beta} d x .
$$

Define

$$
t^{*}=\left(\frac{l(\sigma-q)(\sigma-p)\|(u, v)\|^{\sigma}}{(m-q)(m-p) \int_{\Omega}|u|^{\alpha}|v|^{\beta} d x}\right)^{\frac{1}{m-\sigma}}
$$

It is easy to check that $E(t)$ increases for $t \in\left[0, t^{*}\right)$ and decreases for $t \in\left(t^{*}, \infty\right), E(t)$ achieves its maximum at $t^{*}$. Since $E(t) \rightarrow 0$ as $t \rightarrow 0^{+}$and $E(t) \rightarrow-\infty$ as $t \rightarrow \infty$ and there exists unique $t_{l}, 0<t^{*}<t_{l}$, such that $E\left(t_{l}\right)=0$, so $\eta(t)$ achieves its maximum at $t_{l}$, increasing for $t \in\left[0, t_{l}\right)$ and decreasing for $t \in\left(t_{l}, \infty\right)$. When $l=0$, we have

$$
t_{0}=\left(\frac{k(p-q)\|(u, v)\|^{p}}{(m-q) \int_{\Omega}|u|^{\alpha}|v|^{\beta} d x}\right)^{\frac{q}{m-p}}
$$

Obviously, $E\left(t_{0}\right)=E\left(t_{l}\right)=0$ and $t_{0} \leq t_{l}$ for $l \geq 0$. Thus

$$
\eta\left(t_{l}\right) \geq \frac{k(m-p)}{m-q} t_{l}^{p-q}\|(u, v)\|^{p} \geq \frac{k(m-p)}{m-q} t_{0}^{p-q}\|(u, v)\|^{p}=\eta\left(t_{0}\right)
$$

Set

$$
\begin{aligned}
\Psi_{0}(t) & =\Phi(t u, t v)=\left\langle I^{\prime}(t u, t v)(t u, t v)\right\rangle \\
& =k t^{p}\|(u, v)\|^{p}+l t^{\sigma}\|(u, v)\|^{\sigma}-t^{m} \int_{\Omega}|u|^{\alpha}|v|^{\beta} d x-t^{q} G(u, v), \\
\Psi_{1}(t) & =\left\langle\Phi^{\prime}(t u, t v),(t u, t v)\right\rangle \\
& =k p t^{p}\|(u, v)\|^{p}+l \sigma t^{\sigma}\|(u, v)\|^{\sigma}-m t^{m} \int_{\Omega}|u|^{\alpha}|v|^{\beta} d x-q t^{q} G(u, v) .
\end{aligned}
$$


Then

$$
\Psi_{0}(t)=t^{q}(\eta(t)-G(u, v))
$$

Lemma $2.5(t u, t v) \in N^{+}\left(\right.$or $\left.N^{-}\right)$if and only if $\Psi_{1}(t)>0\left(\right.$ or $\left.\Psi_{1}(t)<0\right)$.

Proof By (2.7), it is clear that $(t u, t v) \in N^{+}$(or $\left.N^{-}\right)$if and only if $(t u, t v) \in N$ and $\left\langle\Phi^{\prime}(t u, t v),(t u, t v)\right\rangle>0(<0)$ for $t>0$. Note that

$$
\Psi_{0}(t)=\Phi(t u, t v)=\left\langle I^{\prime}(t u, t v),(t u, t v)\right\rangle, \quad \Psi_{1}(t)=\left\langle\Phi^{\prime}(t u, t v),(t u, t v)\right\rangle .
$$

Hence, $(t u, t v) \in N^{+}$if and only if $\Psi_{0}(t)=0$ and $\Psi_{1}(t)>0$.

Lemma 2.6 For each $(u, v) \in E \backslash(0,0)$ and $0<\lambda+\mu<\Lambda_{0}$, there exist $0<t_{1}<t_{l}<t_{2}$ such that $\left(t_{1} u, t_{1} v\right) \in N^{+},\left(t_{2} u, t_{2} v\right) \in N^{-}$, and

$$
I\left(t_{1} u, t_{1} v\right)=\inf _{0 \leq t \leq t_{l}} I(t u, t v), \quad I\left(t_{2} u, t_{2} v\right)=\sup _{t \geq 0} I(t u, t v) .
$$

Proof Set

$$
\begin{aligned}
\Psi_{2}(t) & =I(t u, t v) \\
& =\frac{k t^{p}}{p}\|(u, v)\|^{p}+\frac{l t^{\sigma}}{\sigma}\|(u, v)\|^{\sigma}-\frac{t^{m}}{m} \int_{\Omega}|u|^{\alpha}|v|^{\beta} d x-\frac{t^{q}}{q} G(u, v) .
\end{aligned}
$$

Since $0<\lambda+\mu<\Lambda_{0}$, by (2.8), (2.15) and (2.17), we have

$$
G(u, v) \leq(\lambda+\mu)|\Omega|^{\frac{m-q}{m}} S^{-\frac{q}{p}}\|(u, v)\|^{q} \leq \eta\left(t_{0}\right) \leq \eta\left(t_{l}\right) .
$$

Thus, there exist $t_{1}$ and $t_{2}$ such that $0<t_{1}<t_{l}<t_{2}$ and $\eta\left(t_{1}\right)=\eta\left(t_{2}\right)=G(u, v)$. It follows from (2.18) that $\Psi_{0}\left(t_{1}\right)=0$ and $\Psi_{0}\left(t_{2}\right)=0$, then $\left(t_{1} u, t_{1} v\right) \in N$ and $\left(t_{2} u, t_{2} v\right) \in N . \Psi_{1}\left(t_{1}\right)=$ $\left(t_{1}\right)^{q+1} \eta^{\prime}\left(t_{1}\right)>0$. By Lemma 2.5, one has $\left(t_{1} u, t_{1} v\right) \in N^{+}$. Meanwhile, $\Psi_{1}\left(t_{2}\right)=\left(t_{2}\right)^{q+1} \eta^{\prime}\left(t_{2}\right)<$ 0 , we obtain $\left(t_{2} u, t_{2} v\right) \in N^{-}$. By a direct calculation, we have $\Psi_{2}^{\prime}(t)=t^{q-1}(\eta(t)-G(u, v))$. Since $\Psi_{2}^{\prime}(t)<0$ for $t \in\left[0, t_{1}\right)$ and $\Psi_{2}^{\prime}(t)>0$ for $t \in\left[t_{1}, t_{l}\right), I\left(t_{1} u, t_{1} v\right)=\inf _{0 \leq t \leq t_{l}} I(t u, t v)$. Furthermore, we find that $\Psi_{2}^{\prime}(t)>0$ for $t \in\left[t_{1}, t_{2}\right), \Psi_{2}^{\prime}(t)<0$ for $t \in\left[t_{2},+\infty\right)$ and $\Psi_{2}(t) \leq 0$ for $t \in\left[0, t_{1}\right]$. Since $\left(t_{2} u, t_{2} v\right) \in N^{-}$, by Lemma 2.4, we obtain $\Psi_{2}\left(t_{2}\right)>0$. Then $I\left(t_{2} u, t_{2} v\right)=$ $\sup _{t \geq 0} I(t u, t v)$.

\section{Proof of the main result}

Lemma 3.1 If $0<\lambda+\mu<\Lambda_{0}$, then the functional I has a minimizer $\left(u_{1}, v_{1}\right)$ in $N^{+}$satisfying

(i) $I\left(u_{1}, v_{1}\right)=C^{+}<0$;

(ii) $\left(u_{1}, v_{1}\right)$ is a solution of problem (1.1).

Proof Since $I$ is bounded from below on $N^{+}$, there exists a minimizing sequence $\left\{\left(u_{n}\right.\right.$, $\left.\left.v_{n}\right)\right\} \in N^{+}$such that

$$
\lim _{n \rightarrow \infty} I\left(u_{n}, v_{n}\right)=\inf _{(u, v) \in N^{+}} I(u, v)=C^{+} .
$$


Since $I(u, v)$ is coercive and bounded from below on $N$, then $\left\{\left(u_{n}, v_{n}\right)\right\}$ is bounded on $E$. Then there exists $\left(u_{1}, v_{1}\right) \in E$, up to a subsequence, that we still denote by $\left\{\left(u_{n}, v_{n}\right)\right\}$, such that, as $n \rightarrow \infty$,

$$
\begin{aligned}
& u_{n} \rightarrow u_{1}, \quad v_{n} \rightarrow v_{1}, \quad \text { in } L^{r}(\Omega), \\
& u_{n}(x) \rightarrow u_{1}(x), \quad v_{n}(x) \rightarrow v_{1}(x), \quad \text { a.e. in } \Omega
\end{aligned}
$$

for any $1 \leq r<p^{*}$, and by [28], Theorem IV-9, there exists $l(x) \in L^{r}\left(\mathbb{R}^{n}\right)$ such that

$$
\left|u_{n}(x)\right| \leq l(x), \quad\left|v_{n}(x)\right| \leq l(x), \quad \text { a.e. in } \mathbb{R}^{n}
$$

for any $1 \leq r<p^{*}$. By the dominated convergence theorem,

$$
\begin{aligned}
\lim _{n \rightarrow \infty} \int_{\Omega}\left(\lambda\left|u_{n}\right|^{q}+\mu\left|v_{n}\right|^{q}\right) d x & =\int_{\Omega} \lim _{n \rightarrow \infty}\left(\lambda\left|u_{n}\right|^{q}+\mu\left|v_{n}\right|^{q}\right) d x \\
& =\int_{\Omega}\left(\lambda\left|u_{1}\right|^{q}+\mu\left|v_{1}\right|^{q}\right) d x,
\end{aligned}
$$

and

$$
\lim _{n \rightarrow \infty} \int_{\Omega}\left|u_{n}\right|^{\alpha}\left|v_{n}\right|^{\beta} d x=\int_{\Omega}\left|u_{1}\right|^{\alpha}\left|v_{1}\right|^{\beta} d x
$$

By Lemma 2.6, there exists $t_{1}<t_{l}$ such that $\left(t_{1} u_{1}, t_{1} v_{1}\right) \in N^{+}$and $\Psi_{0}\left(t_{1}\right)=\left\langle I^{\prime}\left(t_{1} u_{1}, t_{1} v_{1}\right)\right.$, $\left.\left(t_{1} u_{1}, t_{1} v_{1}\right)\right\rangle=0$.

Next we show that $\left(u_{n}, v_{n}\right) \rightarrow\left(u_{1}, v_{1}\right)$ strongly in $E$. Suppose otherwise, then

$$
\left\|\left(u_{1}, v_{1}\right)\right\|<\liminf _{n \rightarrow \infty}\left\|\left(u_{n}, v_{n}\right)\right\| .
$$

As

$$
\begin{aligned}
\left\langle I^{\prime}\left(t_{1} u_{n}, t_{1} v_{n}\right),\left(t_{1} u_{n}, t_{1} v_{n}\right)\right\rangle= & k t_{1}^{p}\left\|\left(u_{n}, v_{n}\right)\right\|^{p}+l t_{1}^{\sigma}\left\|\left(u_{n}, v_{n}\right)\right\|^{\sigma} \\
& -t_{1}^{m} \int_{\Omega}\left|u_{n}\right|^{\alpha}\left|v_{n}\right|^{\beta} d x-t_{1}^{q} G\left(u_{n}, v_{n}\right),
\end{aligned}
$$

and

$$
\begin{aligned}
\left\langle I^{\prime}\left(t_{1} u_{1}, t_{1} v_{1}\right),\left(t_{1} u_{1}, t_{1} v_{1}\right)\right\rangle= & k t_{1}^{p}\left\|\left(u_{1}, v_{1}\right)\right\|^{p}+l t_{1}^{\sigma}\left\|\left(u_{1}, v_{1}\right)\right\|^{\sigma} \\
& -t_{1}^{m} \int_{\Omega}\left|u_{1}\right|^{\alpha}\left|v_{1}\right|^{\beta} d x-t_{1}^{q} G\left(u_{1}, v_{1}\right),
\end{aligned}
$$

we have

$$
\lim _{n \rightarrow \infty}\left\langle I^{\prime}\left(t_{1} u_{n}, t_{1} v_{n}\right),\left(t_{1} u_{n}, t_{1} v_{n}\right)\right\rangle>\left\langle I^{\prime}\left(t_{1} u_{1}, t_{1} v_{1}\right),\left(t_{1} u_{1}, t_{1} v_{1}\right)\right\rangle=\Psi_{0}\left(t_{1}\right)=0 .
$$

That is, $\left\langle I^{\prime}\left(t_{1} u_{n}, t_{1} v_{n}\right),\left(t_{1} u_{n}, t_{1} v_{n}\right)\right\rangle>0$ for $n$ large enough. Since $\left\{\left(u_{n}, v_{n}\right)\right\} \in N^{+}$, it is easy to see that $\left\langle I^{\prime}\left(u_{n}, v_{n}\right),\left(u_{n}, v_{n}\right)\right\rangle=0$, and $\left\langle I^{\prime}\left(t u_{n}, t v_{n}\right),\left(t u_{n}, t v_{n}\right)\right\rangle<0$ for $0<t<1$. So we have 
$t_{1}>1$. On the other hand, $I\left(t u_{1}, t v_{1}\right)$ is decreasing on $\left(0, t_{1}\right)$, So

$$
I\left(t_{1} u_{1}, t_{1} v_{1}\right) \leq I\left(u_{1}, v_{1}\right)<\liminf _{n \rightarrow \infty} I\left(u_{n}, v_{n}\right)=C^{+}=\inf _{(u, v) \in N^{+}} I(u, v)
$$

which is a contradiction. Hence $\left(u_{n}, v_{n}\right) \rightarrow\left(u_{1}, v_{1}\right)$ strongly in $E$. This implies

$$
I\left(u_{n}, v_{n}\right) \rightarrow I\left(u_{1}, v_{1}\right)=\inf _{(u, v) \in N^{+}} I(u, v)=C^{+} \quad \text { as } n \rightarrow \infty .
$$

Namely, $\left(u_{1}, v_{1}\right)$ is a minimizer of $I$ on $N^{+}$, by Lemma $2.2,\left(u_{1}, v_{1}\right)$ is a solution of problem (1.1).

Lemma 3.2 If $0<\lambda+\mu<\Lambda_{0}$, then the functional I has a minimizer $\left(u_{2}, v_{2}\right)$ in $N^{-}$such that

(i) $I\left(u_{2}, v_{2}\right)=C^{-}$;

(ii) $\left(u_{2}, v_{2}\right)$ is a solution of problem (1.1).

Proof Since $I$ is bounded from below on $N^{-}$, there exists a minimizing sequence $\left\{\left(\bar{u}_{n}\right.\right.$, $\left.\left.\bar{v}_{n}\right)\right\} \in N^{-}$such that

$$
\lim _{n \rightarrow \infty} I\left(\bar{u}_{n}, \bar{v}_{n}\right)=C^{-} .
$$

Since $I(u, v)$ is coercive, $\left\{\left(\bar{u}_{n}, \bar{v}_{n}\right)\right\}$ is bounded on $E$, up to a subsequence, we still denote it by $\left\{\left(\bar{u}_{n}, \bar{v}_{n}\right)\right\}$, then there exists $\left(u_{2}, v_{2}\right) \in E$ such that

$$
\bar{u}_{n} \rightarrow u_{2}, \quad \bar{v}_{n} \rightarrow v_{2}, \quad \text { in } L^{r}(\Omega)
$$

for any $1 \leq r<p^{*}$, and by [28], Theorem IV-9, and the dominated convergence theorem,

$$
\lim _{n \rightarrow \infty} G\left(\bar{u}_{n}, \bar{v}_{n}\right)=G\left(u_{2}, v_{2}\right)
$$

and

$$
\lim _{n \rightarrow \infty} \int_{\Omega}\left|\bar{u}_{n}\right|^{\alpha}\left|\bar{v}_{n}\right|^{\beta} d x=\int_{\Omega}\left|u_{2}\right|^{\alpha}\left|v_{2}\right|^{\beta} d x
$$

By Lemma 2.6, there exists unique $t_{2}$ such that $\left(t_{2} u_{2}, t_{2} v_{2}\right) \in N^{-}$. Next we show that $\left(\bar{u}_{n}, \bar{v}_{n}\right) \rightarrow\left(u_{2}, v_{2}\right)$ strongly in $E$. The proof of this claim is by contradiction. If the claim were not true, then

$$
\left\|\left(u_{2}, v_{2}\right)\right\|<\liminf _{n \rightarrow \infty}\left\|\left(\bar{u}_{n}, \bar{v}_{n}\right)\right\| .
$$

Since $\left(\bar{u}_{n}, \bar{v}_{n}\right) \in N^{-}$and $I\left(\bar{u}_{n}, \bar{v}_{n}\right) \geq I\left(t \bar{u}_{n}, t \bar{v}_{n}\right)$ for all $t>0$, then we have

$$
I\left(t_{2} u_{2}, t_{2} v_{2}\right)<\liminf _{n \rightarrow \infty} I\left(t_{2} \bar{u}_{n}, t_{2} \bar{v}_{n}\right) \leq \liminf _{n \rightarrow \infty} I\left(\bar{u}_{n}, \bar{v}_{n}\right)=C^{-},
$$

which is a contradiction. This implies

$$
I\left(\bar{u}_{n}, \bar{v}_{n}\right) \rightarrow I\left(u_{2}, v_{2}\right)=\inf _{(u, v) \in N^{-}} I(u, v)=C^{-} \quad \text { as } n \rightarrow \infty .
$$


Namely, $\left(u_{2}, v_{2}\right)$ is a minimizer of $I$ on $N^{-}$, by Lemma $2.2,\left(u_{2}, v_{2}\right)$ is a solution of problem (1.1).

Proof of Theorem 1.2 By Lemmas 3.1 and 3.2, we have that for $0<\lambda+\mu<\Lambda_{0}$, problem (1.1) has two solutions $\left(u_{1}, v_{1}\right) \in N^{+}$and $\left(u_{2}, v_{2}\right) \in N^{-}$in $E$. Since $N^{+} \cap N^{-}=\emptyset$, then these two solutions are distinct.

Competing interests

The authors declare that they have no competing interests.

Authors' contributions

All authors contributed equally to the writing of this paper. The authors read and approved the final manuscript.

\section{Acknowledgements}

The authors thank the anonymous referees for invaluable comments and insightful suggestions.

Received: 5 December 2016 Accepted: 18 February 2017 Published online: 22 February 2017

\section{References}

1. Alves, CO, Corrêa, FJSA, Figueiredo, GM: On a class of nonlocal elliptic problems with critical growth. Differ. Equ. Appl. 2, 409-417 (2010)

2. Chen, SJ, Li, L: Multiple solutions for the nonhomogeneous Kirchhoff equation on $R^{n}$. Nonlinear Anal., Real World Appl. 14, 1477-1486 (2013)

3. Figueiredo, GM, Santos, JR Jr:: Multiplicity of solutions for a Kirchhoff equation with subcritical or critical growth Differ. Integral Equ. 25, 853-868 (2012)

4. Figueiredo, GM: Existence of a positive solution for a Kirchhoff problem type with critical growth via truncation argument. J. Math. Anal. Appl. 401, 706-713 (2013)

5. Autuori, G, Pucci, P: Kirchhoff systems with dynamic boundary conditions. Nonlinear Anal. 73, 1952-1965 (2010)

6. Colasuonno, F, Pucci, P: Multiplicity of solutions for $p(x)$-polyharmonic Kirchhoff equations. Nonlinear Anal. 74, 5962-5974 (2011)

7. Molica Bisci, G, Radulescu, V, Servadei, R: Variational Methods for Nonlocal Fractional Problems. Encyclopedia of Mathematics and Its Applications., vol. 162. Cambridge University Press, Cambridge (2016). ISBN:9781107111943

8. Servadei, R, Valdinoci, E: The Brezis-Nirenberg result for the fractional Laplacian. Trans. Am. Math. Soc. 367, 67-102 (2015)

9. Fiscella, A, Valdinoci, E: A critical Kirchhoff type problem involving a nonlocal operator. Nonlinear Anal. 94, 156-170 (2014)

10. Goyal, S, Sreenadh, K: A Nehari manifold for non-local elliptic operator with concave-convex nonlinearities and sign-changing weight functions (2013). arXiv:1307.5149

11. Chen, WJ, Deng, SB: The Nehari manifold for a fractional $p$-Laplacian system involving concave-convex nonlinearities Nonlinear Anal., Real World Appl. 27, 80-92 (2016)

12. Perera, K, Squassina, M, Yang, Y: Critical fractional $p$-Laplacian problems with possibly vanishing potentials. J. Math. Anal. Appl. 433, 818-831 (2016)

13. Goyal, S, Sreenadh, K: Existence of multiple solutions of $p$-fractional Laplace operator with sign-changing weight function. Adv. Nonlinear Anal. 4, 37-58 (2015)

14. Perera, K, Squassina, M, Yang, Y: A note on the Dancer-Fucik spectra of the fractional $p$-Laplacian and Laplacian operators. Adv. Nonlinear Anal. 4, 13-23 (2015)

15. Lehrer, R, Maia, LA, Squassina, M: On fractional $p$-Laplacian problems with weight. Differ. Integral Equ. 28, 15-28 (2015)

16. Bucur, C, Valdinoci, E: Nonlocal Diffusion and Applications. Lecture Notes of the Unione Matematica Italiana, vol. 20. Springer; Unione Matematica Italiana, Bologna (2016). ISBN:978-3-319-28738-6; 978-3-319-28739-3

17. Di Nezza, E, Palatucci, G, Valdinoci, E: Hitchhiker's guide to the fractional Sobolev spaces. Bull. Sci. Math. 136, 521-573 (2012)

18. Dipierro, S, Medina, M, Peral, I, Valdinoci, E: Bifurcation results for a fractional elliptic equation with critical exponent in $R^{n}$. Manuscr. Math. (2016). doi:10.1007/s00229-016-0878-3

19. Drabek, P, Pohozaev, SI: Positive solutions for the $p$-Laplacian: application of the fibering method. Proc. R. Soc. Edinb. A 127, 703-726 (1997)

20. Bozhkov, Y, Mitidieri, E: Existence of multiple solutions for quasilinear systems via fibering method. J. Differ. Equ. 190 239-267 (2003)

21. Ambrosetti, A, Azorero, JG, Peral, I: Multiplicity results for some nonlinear elliptic equations. J. Funct. Anal. 137 219-242 (1996)

22. Hsu, TS, Lin, HL: Multiple positive solutions for a critical elliptic system with concave-convex nonlinearities. Proc R. Soc. Edinb., Sect. A 139, 1163-1177 (2009)

23. Wu, TF: The Nehari manifold for a semilinear elliptic system involving sign-changing weight functions. Nonlinear Anal. 68, 1733-1745 (2008)

24. Servadei, R, Variational, E: Variational methods for non-local operators of elliptic type. Discrete Contin. Dyn. Syst. 33 2105-2137 (2013)

25. Servadei, R, Valdinoci, E: Mountain pass solutions for non-local elliptic operators. J. Math. Anal. Appl. 389, 887-898 (2012) 
26. Autuoria, G, Pucci, P: Elliptic problems involving the fractional Laplacian in $R^{n}$. J. Differ. Equ. 255, 2340-2362 (2013)

27. Xiu, ZH, Chen, CS, Huang, JC: Existence of multiple solution for an elliptic system with sign-changing weight functions. J. Math. Anal. Appl. 395, 531-541 (2012)

28. Brezis, H: Analyse fonctionelle. In: Th'eorie et Applications. Masson, Paris (1983)

Submit your manuscript to a SpringerOpen ${ }^{\circ}$ journal and benefit from:

- Convenient online submission

Rigorous peer review

- Immediate publication on acceptance

- Open access: articles freely available online

- High visibility within the field

Retaining the copyright to your article

Submit your next manuscript at $\boldsymbol{s p r i n g e r o p e n . c o m ~}$ 